\title{
Entropy Message Passing
}

\author{
Velimir M. Ilić, Miomir S. Stanković and Branimir T. Todorović
}

\begin{abstract}
The paper proposes a new message passing algorithm for cycle-free factor graphs. The proposed "entropy message passing" (EMP) algorithm may be viewed as sumproduct message passing over the entropy semiring, which has previously appeared in automata theory. The primary use of EMP is to compute the entropy of a model. However, EMP can also be used to compute expressions that appear in expectation maximization and in gradient descent algorithms.
\end{abstract}

Index Terms-factor graphs, graphical models, sum-product message passing, commutative semiring, entropy, expectation maximization, gradient methods.

\section{INTRODUCTION}

The efficient marginalization of a multivariate function is important in many areas including signal processing, artificial intelligence, and digital communications. When a cycle-free factor graph representation of the function is available, then exact marginals can be computed by sum-product message passing in the factor graph [1]-[4]. In fact, a number of wellknown algorithms are special cases of sum-product message passing.

The "sum" and the "product" in sum-product message passing may belong to an arbitrary commutative semiring [4],[18]. In this paper, we propose to use it with the entropy semiring and the resulting algorithm will be called "entropy message passing" $(E M P)$. The entropy semiring was introduced by Cortes et al. [5] to compute the relative entropy between probabilistic automata. In this paper, we translate the ideas of [5] into the language of factor graphs and message passing algorithms.

The primary use of $E M P$ is to compute the entropy of a model with a cycle-free factor graph for fixed observations. The main prior work on this subject is by Hernando et al. [6]; again, a main point of the present paper is to clarify and to generalize this prior work by reformulating it in terms of sumproduct message passing. However, EMP can also be used to compute expressions that appear in expectation maximization and in gradient ascent algorithms [7]-[13]; this connection appears to be new.

The paper is structured as follows. In Section II, we review sum-product message passing over a commutative semiring. In Section III, we introduce the entropy semiring. The EMP algorithm is described in Section IV and the mentioned applications are described in Section V.

V. Ilić is with the Department of Informatics, Faculty of Sciences and Mathematics, University of Niš, Serbia,

M. Stanković is with the Faculty of Occupational Safety, University of Niš, Serbia,

B. Todorovic is with the Department of Informatics, Faculty of Sciences and Mathematics, University of Niš, Serbia.

\section{FACTOR GRAPHS AND THE Sum-Product ALGORITHM}

Let $f$ be a real multivariate function that depends on the set of variables $\mathbf{x}=\{x\}_{n=1}^{N}$ and satisfies

$$
f(\mathbf{x})=\prod_{m \in \mathcal{M}} f_{m}\left(\mathbf{x}_{m}\right)
$$

for a set of indexes $\mathcal{M}$. In the expression (1) each factor $f_{m}\left(\mathbf{x}_{m}\right)$ depends on $\mathbf{x}_{m} \subset \mathbf{x}$ and the subsets $\mathbf{x}_{m}$ cover $\mathbf{x}$. The factorization (1) can be graphically represented by a factor graph [1]-[3]. A factor graph consists of the variable nodes (drawn as circles), the factor nodes (drawn as squares) and the connections between the nodes, where the variable node $n$ and the factor node $m$ are connected if and only if the factor $f_{m}$ depends on the variable $x_{n}$. An example of a factor graph is given in Fig. 1.

The following two problems are of interest in many applications [14]:

1) The marginalization problem:

$$
Z_{n}\left(x_{n}\right)=\sum_{\mathbf{x} \backslash x_{n}} \prod_{m \in \mathcal{M}} f_{m}\left(\mathbf{x}_{m}\right),
$$

where $\sum_{\mathbf{x} \backslash x_{n}}$ denotes the summing all variables from $\mathbf{x}$ except $x_{n}$ and

2) The normalization problem:

$$
Z=\sum_{\mathbf{x}} \prod_{m \in \mathcal{M}} f_{m}\left(\mathbf{x}_{m}\right) .
$$

The solution of the second problem can easily be obtained from the solution of the first problem by means of:

$$
Z=\sum_{x_{n}} Z_{n}\left(x_{n}\right)
$$

therefore, in the following paragraphs, we are concerned with the solution of the first problem.

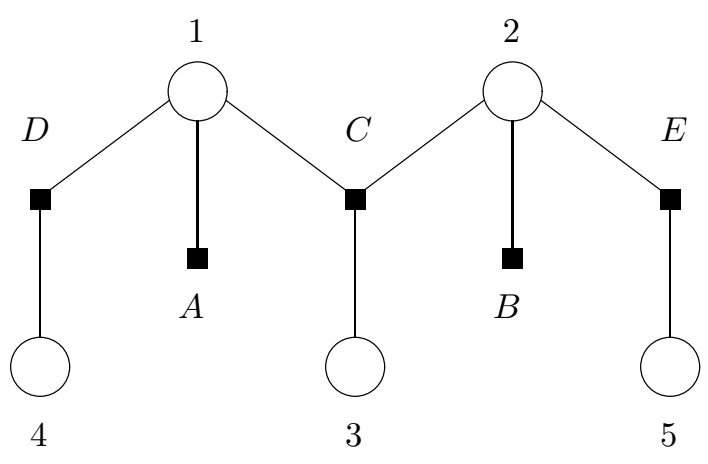

Fig. 1. The factor graph that corresponds to the factorization $f_{A}\left(x_{1}\right) f_{B}\left(x_{2}\right) f_{C}\left(x_{1}, x_{2}, x_{3}\right) f_{D}\left(x_{1}, x_{4}\right) f_{E}\left(x_{2}, x_{5}\right)$. 
The marginalization problem (2) can efficiently been solved using the sum-product algorithm $(S P A)[1]-[3]$. The sumproduct algorithm operates by passing messages along the edges of the factor graph of a function to be marginalized. The computed marginal value is exact for a cycle-free factor graph, but the algorithm can also be applied on graphs with cycles, in which case an approximate solution may be obtained [15]-[17]. In this paper, we consider only cycle-free (i.e., tree structured) factor graphs.

There are two types of messages:

1) the messages $q_{n \rightarrow m}\left(x_{n}\right)$ from variable to factor nodes and

2) the messages $r_{m \rightarrow n}\left(x_{n}\right)$ from factor to variable nodes, where the variable and factor nodes participating in the message passing process are denoted with $n$ and $m$. Note that both types of messages are functions of the variable that is represented by the involved node.

The messages are initialized to $q_{n \rightarrow m}\left(x_{n}\right)=1$ and $r_{m \rightarrow n}\left(x_{n}\right)=f_{m}\left(x_{n}\right)$, for all variable nodes $n$ and factor nodes $m$ in the leaves of the factor graph, for all possible values $x_{n}$. After that the messages are passed toward the root that corresponds to the variable for which the marginal value is computed. The message from a node to its parent is computed after the messages from all descendants are received, according to the following rules:

$$
q_{n \rightarrow m}\left(x_{n}\right)=\prod_{m^{\prime} \in \mathcal{N}(n) \backslash m} r_{m^{\prime} \rightarrow n}\left(x_{n}\right)
$$

and

$$
r_{m \rightarrow n}\left(x_{n}\right)=\sum_{\mathbf{x}_{m} \backslash x_{n}} f_{m}\left(\mathbf{x}_{m}\right) \prod_{n^{\prime} \in \mathcal{N}(m) \backslash n} q_{n^{\prime} \rightarrow m}\left(x_{n^{\prime}}\right) .
$$

Here, $\mathcal{N}(n) \backslash m$ denotes all the nodes that are neighbors of the node $n$ except for the node $m$, and $\sum_{\mathbf{x}_{m} \backslash x_{n}}$ denotes a sum over all the variables $\mathbf{x}_{m}$ that are arguments of $f_{m}$ except $x_{n}$. The process is terminated at the root, where the marginal function is computed according to:

$$
Z_{n}\left(x_{n}\right)=\prod_{m \in \mathcal{N}(n)} r_{m \rightarrow n}\left(x_{n}\right) .
$$

So far, we have implicitly assumed that the function to be marginalized has as codomain the set of real numbers obtained with the standard operations + and $\times$. Nevertheless, the algorithm still works when the codomain is an commutative semiring (see the next section for the definition). The generalized form of the algorithm can be obtained straightforwardly by replacing the operations + and $\times$ from the set of real numbers with the operations $\oplus$ and $\otimes$ from the semiring [1],[4],[18].

\section{The Entropy SEMIRING}

In this section we introduce the algebraic notions that will be useful for development of the EMP algorithm.

Definition 1 [19]: The system $\langle\mathcal{K}, \oplus, \otimes, \overline{0}, \overline{1}\rangle$ is called a commutative semiring if:

1) The operations $\oplus$ and $\otimes$ are associative and commutative;
2) The equalities $k \oplus \overline{0}=k$ and $k \otimes \overline{1}=k$ hold for all $k \in \mathcal{K}$

3) The operation $\otimes$ distributes over $\oplus$, i.e., for all $a, b, c \in$ $\mathcal{K}$ the following equalities hold:

$$
\begin{aligned}
& (a \oplus b) \otimes c=(a \otimes c) \oplus(b \otimes c), \\
& c \otimes(a \oplus b)=(c \otimes a) \oplus(c \otimes b) .
\end{aligned}
$$

Some common commutative semirings are the sumproduct semiring $\left\langle\mathcal{R}_{+},+, \times, 0,1\right\rangle$, the Boolean semiring $\langle\{0,1\}, \wedge, \vee, 0,1\rangle$ and the max-product semiring $\left\langle\mathcal{R}_{+}, \max , \times, 0,1\right\rangle$, where $\mathcal{R}$ denotes the set of real numbers. Other semirings used in the message passing algorithms can be found in [4]. In this paper we consider the entropy semiring [5] (also called the expectation semiring [20]).

Definition 2 The entropy semiring is a tuple

$$
\left\langle\mathcal{R}^{2}, \oplus, \otimes,(0,0),(1,0)\right\rangle,
$$

where the operations $\oplus$ and $\otimes$ are defined with:

$$
\begin{gathered}
\left(x_{1}, y_{1}\right) \oplus\left(x_{2}, y_{2}\right)=\left(x_{1}+x_{2}, y_{1}+y_{2}\right) ; \\
\left(x_{1}, y_{1}\right) \otimes\left(x_{2}, y_{2}\right)=\left(x_{1} x_{2}, x_{1} y_{2}+x_{2} y_{1}\right),
\end{gathered}
$$

for all $\left(x_{1}, y_{1}\right),\left(x_{2}, y_{2}\right) \in \mathcal{R}^{2}$.

The following lemma will be useful for the derivation of the EMP algorithm in the next section.

Lemma 1: Let $\mathcal{M}$ be a finite set of indices. Then, for all $\left(a_{m}, b_{m}\right) \in \mathcal{R}^{2}, m \in \mathcal{M}$ the following equality holds:

$$
\bigotimes_{m \in \mathcal{M}}\left(a_{m}, b_{m}\right)=\left(\prod_{m \in \mathcal{M}} a_{m}, \sum_{m \in \mathcal{M}} b_{m} \prod_{j \in \mathcal{M} \backslash\{m\}} a_{j}\right) .
$$

Proof: We prove the lemma by induction over the cardinality of $\mathcal{M}$. Without loss of generality, suppose that the sets $\mathcal{M}$ have the form $\{1,2, \ldots, k\}$ where $k$ is from the set of the natural numbers. If $\mathcal{M}$ has two elements, the equality (12) reduces to the definition for multiplying in an entropy semiring:

$$
\left(a_{1}, b_{1}\right) \otimes\left(a_{2}, b_{2}\right)=\left(a_{1} a_{2}, a_{1} b_{2}+a_{2} b_{1}\right) .
$$

Now, let the equality hold for some $k$ - element set $\mathcal{M}_{k}=$ $\{1,2, \ldots, k\}$ :

$$
\bigotimes_{m \in \mathcal{M}_{k}}\left(a_{m}, b_{m}\right)=\left(\prod_{m \in \mathcal{M}_{k}} a_{m}, \sum_{m \in \mathcal{M}_{k}} b_{m} \prod_{j \in \mathcal{M}_{k} \backslash\{m\}} a_{j}\right) .
$$

Using this, and using the equality $\mathcal{M}_{k+1}=\mathcal{M}_{k} \cup\{k+1\}$, it is easy to obtain (12) for $k+1$ - element set $\mathcal{M}_{k+1}=$ $\{1,2, \ldots, k+1\}$ :

$$
\bigotimes_{m \in \mathcal{M}_{k+1}}\left(a_{m}, b_{m}\right)=\bigotimes_{m \in \mathcal{M}_{k}}\left(a_{m}, b_{m}\right) \otimes\left(a_{m+1}, b_{m+1}\right)=
$$

$\left(\prod_{m \in \mathcal{M}_{k+1}} a_{m}, b_{k+1} \prod_{m \in \mathcal{M}_{k}} a_{m}+\sum_{m \in \mathcal{M}_{k}} b_{m} \prod_{j \in \mathcal{M}_{k+1} \backslash\{m\}} a_{j}\right)$ 


$$
=\left(\prod_{m \in \mathcal{M}_{k+1}} a_{m}, \sum_{m \in \mathcal{M}_{k+1}} b_{m} \prod_{j \in \mathcal{M}_{k+1} \backslash\{m\}} a_{j}\right),
$$

which proves the lemma.

\section{The Entropy Message Passing Algorithm}

Let $w(\mathbf{x})$ be a multivariate function whose codomain is an entropy semiring $\mathcal{K}$ and let the factorization

$$
w(\mathbf{x})=\bigotimes_{m \in \mathcal{M}} w_{m}\left(\mathbf{x}_{\mathbf{m}}\right)
$$

hold for a set of indices $\mathcal{M}$, where each factor $w_{m}\left(\mathbf{x}_{m}\right)$ depends on $\mathbf{x}_{m} \subset \mathbf{x}$ and subsets $\mathbf{x}_{m}$ cover $\mathbf{x}$. Further, let the factors have a form

$$
w_{m}\left(\mathbf{x}_{m}\right)=\left(f_{m}\left(\mathbf{x}_{m}\right), f_{m}\left(\mathbf{x}_{m}\right) g_{m}\left(\mathbf{x}_{m}\right)\right),
$$

where $f_{m}\left(\mathbf{x}_{m}\right)$ and $g_{m}\left(\mathbf{x}_{m}\right)$ are real functions which depend on the same set of variables $\mathbf{x}_{m}$. Using (12) it is easy to obtain:

$$
w(\mathbf{x})=\left(\prod_{m \in \mathcal{M}} f_{m}\left(\mathbf{x}_{m}\right), \prod_{m \in \mathcal{M}} f_{m}\left(\mathbf{x}_{m}\right) \sum_{k \in \mathcal{M}} g_{k}\left(\mathbf{x}_{k}\right)\right) .
$$

Hence, if a function has the form (15), then it can be factorized as in (13) with factors as in (14).

With a fast computation of

$$
(Z, H)=\bigoplus_{\mathbf{x}} w(\mathbf{x})
$$

we solve two problems:

1) The computation of the expression:

$$
Z=\sum_{\mathbf{x}} \prod_{m \in \mathcal{M}} f_{m}\left(\mathbf{x}_{m}\right)
$$

which is the normalization problem considered in section II and

2) The computation of the expression:

$$
H=\sum_{\mathbf{x}} \prod_{m \in \mathcal{M}} f_{m}\left(\mathbf{x}_{m}\right) \sum_{k \in \mathcal{M}} g_{k}\left(\mathbf{x}_{k}\right)
$$

which is the general form of the different problems described in the next section. These problems are the key motivation for our work.

If the factor graph corresponding to $w(\mathbf{x})$ has a tree structure, the computation (16) can be performed by message passing over the entropy semiring. Note that the factor graph of $w(\mathbf{x})$ has the same topology as the function

$$
f(\mathbf{x})=\prod_{m \in \mathcal{M}} f_{m}\left(\mathbf{x}_{m}\right)
$$

in particular, the factor graph of $w(\mathbf{x})$ is cycle free if and only if the factor graph of $f(\mathbf{x})$ is cycle free. To perform the summation (16), we follow the procedure from section II - we calculate the marginal:

$$
W_{n}\left(x_{n}\right)=\bigoplus_{\mathbf{x} \backslash x_{n}} w(\mathbf{x})
$$

for a variable $x_{n}$, and subsequently we obtain the total sum by:

$$
\bigoplus_{\mathbf{x}} w(\mathbf{x})=\bigoplus_{x_{n}} W_{n}\left(x_{n}\right)
$$

In the following paragraphs, we formalize the discussion by using the Entropy Message Passing (EMP) algorithm:

3) Initialization: Set the messages from all variable and factor nodes in leaves to:

$$
\begin{gathered}
q_{n \rightarrow m}\left(x_{n}\right)=(1,0), \\
r_{m \rightarrow n}\left(x_{n}\right)=\left(f_{m}\left(x_{n}\right), f_{m}\left(x_{n}\right) g_{m}\left(x_{n}\right)\right) .
\end{gathered}
$$

4) Induction: After receiving the messages from all descendants, compute the messages to the parents for all variable and factor nodes in the tree:

$$
\begin{gathered}
q_{n \rightarrow m}\left(x_{n}\right)=\bigotimes_{m^{\prime} \in \mathcal{N}(n) \backslash m} r_{m^{\prime} \rightarrow n}\left(x_{n}\right), \\
r_{m \rightarrow n}\left(x_{n}\right)=\bigoplus_{\mathbf{x}_{m} \backslash x_{n}}\left(f_{m}\left(\mathbf{x}_{m}\right), f_{m}\left(\mathbf{x}_{m}\right) g_{m}\left(\mathbf{x}_{m}\right)\right) \\
\bigotimes_{n^{\prime} \in \mathcal{N}(m) \backslash n} q_{n^{\prime} \rightarrow m}\left(x_{n^{\prime}}\right) .
\end{gathered}
$$

5) Termination: At the root, compute the marginal value and the total sum:

$$
\begin{gathered}
\left(Z_{n}\left(x_{n}\right), H_{n}\left(x_{n}\right)\right)=\bigotimes_{m \in \mathcal{N}(n)} r_{m \rightarrow n}\left(x_{n}\right), \\
(Z, H)=\bigoplus_{x_{n}}\left(Z_{n}\left(x_{n}\right), H_{n}\left(x_{n}\right)\right) .
\end{gathered}
$$

The EMP has the same asymptotic computational complexity as the $S P A$, since addition and multiplication in an entropy semiring are realized via addition and multiplication of the real numbers. The precise complexity estimates of the message passing algorithms can be found in [4].

Note that in the first component, the entropy semiring works like ordinary addition and multiplication; in consequence, EMP (i.e., sum-product message passing over the entropy semiring) contains ordinary sum-product message passing in the first component.

\section{Applications}

In this section we show how EMP applies to the entropy computation and the optimization techniques such as expectation maximization and gradient ascent algorithm.

\section{A. Entropy computation of a partially observed probabilistic model}

In this section we show how EMP can be used for an efficient computation of the state sequence entropy of the partially observed probabilistic models. The algorithm for such computation has previously been proposed in [6], but only for the chain structured models. Applying the EMP, we can generalize this algorithm to the arbitrary probabilistic model the factor graph of which has no cycles. 
Let a partially observed model be given with the probability distribution $P(\mathbf{x}, \mathbf{y})$, where $\mathbf{x}=\left\{x_{1}, \ldots, x_{n}\right\}$ denotes a hidden variable sequence of length $n$ and $\mathbf{y}=\left\{y_{1}, \ldots, y_{m}\right\}$ denotes an observation sequence of length $m$. The entropy of the model $P(\mathbf{x}, \mathbf{y})$ for the given sequence of the observation is given with:

$$
H(X \mid Y=\mathbf{y})=-\sum_{\mathbf{x}} P(\mathbf{x} \mid \mathbf{y}) \log _{2} P(\mathbf{x} \mid \mathbf{y}) .
$$

By use of the Bayes theorem and the additivity of a logarithm, this expression can be transformed to:

$$
\begin{aligned}
& H(X \mid Y=\mathbf{y})= \\
& -\frac{\sum_{\mathbf{x}} P(\mathbf{x}, \mathbf{y}) \log _{2} P(\mathbf{x}, \mathbf{y})}{\sum_{\mathbf{x}} P(\mathbf{x}, \mathbf{y})}+\log _{2} \sum_{\mathbf{x}} P(\mathbf{x}, \mathbf{y}) .
\end{aligned}
$$

Note that the probability distribution $P(\mathbf{x}, \mathbf{y})$ can be considered as a function depending only on the vector variable $\mathbf{x}$, since $\mathbf{y}$ is observed and can be treated as a constant.

Let $P(\mathbf{x}, \mathbf{y})$ factorize as

$$
P(\mathbf{x}, \mathbf{y})=\prod_{m \in \mathcal{M}} f_{m}\left(\mathbf{x}_{m}\right),
$$

that corresponds to a cycle-free factor graph. If $g_{m}\left(\mathbf{x}_{m}\right)=$ $\log _{2} f_{m}\left(\mathbf{x}_{m}\right)$, the expression (29) can be written as:

$$
H(X \mid Y=\mathbf{y})=-\frac{H}{Z}+\log _{2} Z,
$$

where

$$
Z=\sum_{\mathbf{x}} \prod_{m \in \mathcal{M}} f_{m}\left(\mathbf{x}_{m}\right)
$$

and

$$
H=\sum_{\mathbf{x}} \prod_{m \in \mathcal{M}} f_{m}\left(\mathbf{x}_{m}\right) \sum_{k \in \mathcal{M}} g_{k}\left(\mathbf{x}_{k}\right) .
$$

The previous expressions have the form (17) and 18 and can be computed with the EMP, which solves the problem of efficient computation of the model entropy.

\section{B. Iterative optimization techniques}

Suppose we wish to find

$$
\hat{\Theta}_{\max }=\arg \max _{\Theta} p(\Theta)
$$

with a parameter $\Theta$ taking values from $\mathcal{R}$ or $\mathcal{R}^{k}$. We assume that $p(\Theta)$ is the marginal of a real-valued nonnegative function $p(\mathbf{x}, \Theta)$ :

$$
p(\Theta)=\sum_{\mathbf{x}} p(\mathbf{x}, \Theta) .
$$

In this section we consider two popular procedures for solving the problem (34) - the Expectation Maximization (EM) and the gradient ascent algorithm. Both algorithms seek the solution iteratively with the parameter $\Theta$ being estimated in each iteration. In the following paragraphs we show how EMP can be used for the computations which appear here. We suppose that if

$$
p(\mathbf{x}, \Theta)=\prod_{m \in \mathcal{M}} p_{m}\left(\mathbf{x}_{m}, \Theta\right)
$$

is considered as the function of $\mathbf{x}$ only with $\Theta$ fixed, its factor graph is a tree, similarly as in the previous papers which consider the $E M$ algorithm from the message passing point of a view [7]-[10].

1) The expectation maximization algorithm: The EM algorithm [3], [10] attempts to compute (34) as follows:

1) Choose an initial setting for the parameters $\Theta^{\text {old }}$.

2) E-step: Evaluate $p\left(\mathbf{x}, \Theta^{\text {old }}\right)$.

3) $M$-step: Evaluate $\Theta^{\text {new }}$ given by

$$
\Theta^{\text {new }}=\arg \max _{\Theta} Q\left(\Theta, \Theta^{\text {old }}\right)
$$

where

$$
Q\left(\Theta, \Theta^{\text {old }}\right)=\sum_{\mathbf{x}} p\left(\mathbf{x}, \Theta^{\text {old }}\right) \log p(\mathbf{x}, \Theta) .
$$

4) While the convergence criterion is not satisfied, let $\Theta^{\text {old }}=\Theta^{\text {new }}$ and return to step 2 .

The $M$-step is usually performed by solving the equation

$$
\nabla_{\Theta} Q\left(\Theta, \Theta^{\text {old }}\right)=0
$$

where $\nabla_{\Theta}$ denotes the gradient operator. After substituting (36) in (38), the expression (39) can be transformed into

$$
\sum_{\mathbf{x}} \prod_{m \in \mathcal{M}} p_{m}\left(\mathbf{x}_{m}, \Theta^{\text {old }}\right) \sum_{k \in \mathcal{M}} \nabla_{\Theta} \log p_{k}\left(\mathbf{x}_{k}, \Theta\right)=0 .
$$

It can be shown that the $E M$ algorithm always leads to a solution. Nevertheless, it becomes computationally demanding as the number of the steps required for its convergence and dimensionality of $\mathbf{x}$ grow. Yet, this problem can efficiently be solved with the EMP when gradients of the logarithms of the factors in (36) linearly depend on $\Theta$, i.e.

$$
\nabla_{\Theta} \log p_{k}\left(\mathbf{x}_{k}, \Theta\right)=u_{k}\left(\mathbf{x}_{k}\right) \cdot \Theta+v_{k}\left(\mathbf{x}_{k}\right) \cdot \Lambda
$$

where $\Lambda$ is a constant vector of the same dimensionality as $\Theta$ (see [8], [11] and [21] for the examples). Accordingly, the solution of (40) has the form:

$$
\Theta=-\frac{H_{a}}{H_{b}} \cdot \Lambda
$$

where

$$
H_{a}=\sum_{\mathbf{x}} \prod_{m \in \mathcal{M}} p_{m}\left(\mathbf{x}_{m}, \Theta^{\text {old }}\right) \sum_{k \in \mathcal{M}} u_{k}\left(\mathbf{x}_{k}\right)
$$

and

$$
H_{b}=\sum_{\mathbf{x}} \prod_{m \in \mathcal{M}} p_{m}\left(\mathbf{x}_{m}, \Theta^{\text {old }}\right) \sum_{k \in \mathcal{M}} v_{k}\left(\mathbf{x}_{k}\right) .
$$

The expressions for $H_{a}$ and $H_{b}$ can efficiently be computed with the EMP algorithm since both can be derived from (18) by the settings $f_{m}\left(\mathbf{x}_{m}\right)=p_{m}\left(\mathbf{x}_{m}, \Theta^{\text {old }}\right)$ and $g_{k}\left(\mathbf{x}_{k}\right)=u_{k}\left(\mathbf{x}_{k}\right)$ for $H_{a}$ and $f_{m}\left(\mathbf{x}_{m}\right)=p_{m}\left(\mathbf{x}_{m}, \Theta^{\text {old }}\right)$ and $g_{k}\left(\mathbf{x}_{k}\right)=v_{k}\left(\mathbf{x}_{k}\right)$ for $H_{b}$. 
2) The gradient ascent algorithm: The previously described procedure for parameter estimation can be applied when the linear dependence (41) holds, but when the dependency is nonlinear, the analytic solution for the $M$-step does not exist in general. Instead, we can apply the gradient ascent algorithm [11]-[13] to solve the optimization problem (34). The gradient ascent seeks the maximum of real nonnegative differentiable function $p(\Theta)$ by an iterative process:

$$
\Theta_{i+1}=\Theta_{i}+\left.\nabla_{\Theta} p(\Theta)\right|_{\Theta_{i}}
$$

where $\left.\nabla_{\Theta} p(\Theta)\right|_{\Theta_{i}}$ denotes the gradient of $p(\Theta)$ at the point $\Theta_{i}$. Since $p(\Theta)$ is given by the marginal (35), the gradient can be written as

$$
\left.\nabla_{\Theta} p(\Theta)\right|_{\Theta_{i}}=\left.\sum_{\mathbf{x}} \nabla_{\Theta} p(\mathbf{x}, \Theta)\right|_{\Theta_{i}} .
$$

If we apply Leibniz's rule to the factorization (36), the previous expression becomes:

$$
\left.\nabla_{\Theta} p(\Theta)\right|_{\Theta_{i}}=\sum_{\mathbf{x}} \prod_{m \in \mathcal{M}} f_{m}\left(\mathbf{x}_{m}\right) \sum_{k \in \mathcal{M}} g_{k}\left(\mathbf{x}_{k}\right),
$$

where

$$
f_{m}\left(\mathbf{x}_{m}\right)=p_{m}\left(\mathbf{x}_{m}, \Theta_{i}\right)
$$

and

$$
g_{k}\left(\mathbf{x}_{k}\right)=\left.\frac{\nabla_{\Theta} p_{k}\left(\mathbf{x}_{k}, \Theta\right)}{p_{k}\left(\mathbf{x}_{k}, \Theta\right)}\right|_{\Theta=\Theta_{i}} .
$$

Again, we have the expression of the form [18), so the gradient (47) can be evaluated with the EMP algorithm.

The gradient ascent can also be used for the $M$-step of the $E M$ algorithm as in [12], [22] and [23]. In this case, 338 should be maximized by the iterative procedure:

$$
\Theta_{i+1}=\Theta_{i}+\left.\nabla_{\Theta} Q\left(\Theta, \Theta^{\text {old }}\right)\right|_{\Theta_{i}} .
$$

The computation that appears here can also be performed with the $E M P$, since the gradient of $Q\left(\Theta, \Theta^{\text {old }}\right)$ reduces to (47) for

$$
f_{m}\left(\mathbf{x}_{m}\right)=p_{m}\left(\mathbf{x}_{m}, \Theta^{\text {old }}\right)
$$

and

$$
g_{k}\left(\mathbf{x}_{k}\right)=\left.\frac{\nabla_{\Theta} p_{k}\left(\mathbf{x}_{k}, \Theta\right)}{p_{k}\left(\mathbf{x}_{k}, \Theta\right)}\right|_{\Theta=\Theta_{i}},
$$

which can easily be shown.

\section{CONCLUSION}

Building on previous work [5], [6], we have proposed a new general message passing algorithm for factor graphs, which we call entropy message passing $(E M P)$. EMP may be viewed as a new version of sum-product message passing over the entropy semiring. The following applications of EMP have been demonstrated: 1) the computation of the entropy of an observed hidden Markov model with fixed observations (in this application, EMP is essentially the algorithm of [5]),2) expectation maximization and 3) gradient-ascent algorithms.

As a version of sum-product message passing, EMP gives exact results only for factor graphs without cycles. Nevertheless, the algorithm can be applied (without guarantees) also to factor graphs with cycles, where it might give good empirical results in some cases.

\section{ACKNOWLEDGMENT}

The authors would like to thank thank the anonymous reviewers for their valuable comments and suggestions that improved the presentation of this paper.

\section{REFERENCES}

[1] F. R. Kschischang, B. J. Frey, and H.-A. Loeliger, "Factor graphs and the sum-product algorithm," IEEE Trans. Inform. Theory, vol. 47, pp. 498-519, 2001.

[2] H.-A. Loeliger, "An introduction to factor graphs," IEEE Signal Proc. Mag., pp. 28-41, Jan. 2004.

[3] C. M. Bishop, Pattern Recognition and Machine Learning. New York, Springer, 2006.

[4] S. M. Aji and R. J. McEliece, "The generalized distributive law," IEEE Trans. Inform. Theory, vol. 46, pp. 325-343, Mar. 2000.

[5] C. Cortes, M. Mohri, A. Rastogi and M. Riley, "On the computation of the relative entropy of probabilistic automata," International Journal of Foundations of Computer Science, vol. 19, pp. 219-242, 2007.

[6] D. Hernando, V. Crespi and G. Cybenko, "Efficient computation of the Hidden Markov model entropy for a given observation sequence," IEEE Trans. Inform. Theory, vol. 51, pp. 2681-2687, July 2005.

[7] A. W. Eckford and S. Pasupathy, "Iterative multiuser detection with graphical modeling," in Proc. IEEE International Conference on Personal Wireless Communications, Hyderabad,India, pp. 454-458, 2000.

[8] A. W. Eckford, "Channel estimation in block fading channels using the factor graph EM algorithm," in Proc. 22nd Biennial Symposium on Communications, Kingston, ON, Canada, pp. 44-46, 2004.

[9] J. Dauwels, S. Korl, and H.-A. Loeliger, "Expectation maximization as message passing," in Proc. 2005 IEEE Int. Symp. Information Theory, Adelaide, Australia, Sep. 4-9, 2005, pp. 583-586.

[10] J. Dauwels, A. Eckford, S. Korl, and H.-A. Loeliger, "Expectation maximization as message passing - Part I: Principles and Gaussian Messages," arXiv:0910.2832 Oct. 2009.

[11] H.-A. Loeliger, J. Dauwels, Junli Hu, S. Korl, Li Ping, and F. R. Kschischang, "The factor graph approach to model-based signal processing," Proceedings of the IEEE, vol. 95, no. 6, pp. 1295-1322, June 2007.

[12] J. Dauwels, S. Korl, and H.-A. Loeliger, "Steepest descent on factor graphs," in Proc. IEEE Information Theory Workshop, Rotorua, New Zealand, Aug. Sep 28, 2005, pp. 42-46.

[13] H.-A. Loeliger, "Some remarks on factor graphs," in Proc. 3rd Int. Symp. on Turbo Codes and Related Topics, Sept. 15, 2003, Brest, France, pp. 111-115.

[14] D. J. C. MacKay, Information Theory, Inference and Learning Algorithms. Cambridge University Press, 2003.

[15] J. S. Yedidia, W. T. Freeman, and Y. Weiss, "Constructing free energy approximations and generalized belief propagation algorithms," IEEE Trans. Inform. Theory, vol. 51, pp. 2282-2312, July 2005.

[16] K.P. Murphy, Y. Weiss, and M.I. Jordan, 'Loopy belief propagation for approximate inference: an empirical study," in Proc. Uncertainty in AI, 1999.

[17] Y. Weiss, "Correctness of local probability propagation in graphical models with loops," Neural Computation, 12, pp. 141, 2000.

[18] N. Wiberg, "Codes and decoding on general graphs," Ph.D. dissertation, Linkoping Univ., Linkoping, Sweden, 1996.

[19] W. Kuich and A. Salomaa, Semirings, Automata, Languages, Number 5 in EATCS Monographs on Theoretical Computer Science, SpringerVerlag, Berlin, Germany, 1986.

[20] J. Eisner, "Parameter estimation for probabilistic finite-state transducers," in Proceedings of the 40th Annual Meeting of the Association for Computational Linguistics, Philadelphia, July 2002, pp. 18.

[21] O. Ronen, J. R. Rohlicek, and M. Ostendorf, "Parameter estimation of dependence tree models using the EM algorithm," IEEE Signal Processing Lett., Aug. 1995.

[22] E. Weinstein, M. Feder, and A. V. Oppenheim, "Sequential algorithms for parameter estimation based on the Kullback-Leibler information measure," IEEE Transactions on Acoustics, Speech, and Signal Processing, vol. 38, pp. 1652-1654, Sept. 1990.

[23] V. Krishnamurthy and J. B. Moore, "On-line estimation of hidden Markov model parameters based on the Kullback-Leibler information measure," IEEE Trans. Signal Processing, vol. 41, pp. 2557-2573, Aug. 1993. 